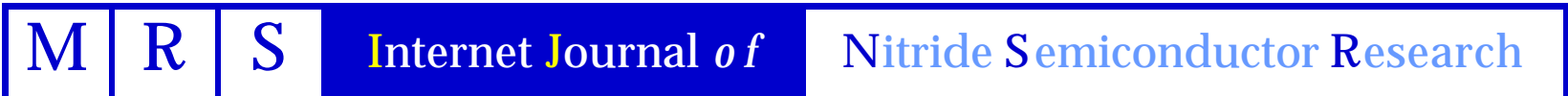

Volume 2, Article 21

\section{Optical nonlinearities of Gallium Nitride}

\author{
H. Haag, P. Gilliot, D. Ohlmann, R. Levy \\ Groupe d'Optique Non Lineaire et d'Optoélectronique,Institut de Physique et Chimie de Strasbourg \\ Olivier Briot, Roger-Louis Aulombard \\ Groupe d'Etude des Semiconducteurs, GES-CNRS
}

This article was received on June 10, 1997 and accepted on August 28, 1997.

\begin{abstract}
Luminescence, induced absorption and degenerate four-wave mixing experiments are perform on GaN epilayers grown on a sapphire substrate by MOCVD.

We measure the nonlinear behavior of the luminescence spectra near the excitonic resonance, by using an excitation at $4.026 \mathrm{eV}$ from an excimer laser. At low intensities of excitation, spectra show a saturation of the 12 line due to the finite donor density in the sample. Higher intensities of excitation induce collision processes between photo-created quasi-particles.

Using a dye laser as a pump beam, we measure the induced variation of absorption of a probe beam as a function of the intensity and of the wavelength of the excitation. With increasing intensities of the pump beam, transmission spectra show a red-shift of the absorption edge and of the excitonic resonance.

Pulsed degenerate four-wave mixing experiments were performed using the third harmonic of a picosecond Nd-YAG laser at $3.492 \mathrm{eV}$. A characteristic time constant of 16 ps has been measured, which is independent of the temperature, spacing of the interference fringe and of the intensity of the pump beams.

The scope of this work is to study optical properties of GaN epilayers near the excitonic region. Our samples are thin layers of GaN grown on a (0001) sapphire substrate by MOCVD, with a buffer layer of GaN. Samples are characterized by linear reflection, transmission and luminescence measurements. We perform luminescence, induced absorption and degenerate four-wave mixing experiments to study the optical nonlinearities of the material.
\end{abstract}

\section{Introduction}

\section{Linear properties}

Linear optical studies allow us to determine the basic properties of our samples. They are placed in a fused silica cryostat and cooled down to $1.8 \mathrm{~K}$.

The luminescence spectra of GaN generally display the radiative recombination of excitons bound to neutral donors ( $\mathrm{I}_{2}$ line), but one of our high quality samples, of $1.8 \mu \mathrm{m}$ thickness, exhibits three emission lines at photon energies of $3.474,3.480$ and $3.486 \mathrm{eV}$. They are attributed to the $\mathrm{I}_{2}, \mathrm{~A}$ and $\mathrm{B}$ exciton lines $\left(\mathrm{X}_{\mathrm{A}}\right.$ and $\mathrm{X}$ $B)$ respectively [1]. The nonlinear luminescence of this sample will be described in the next section. 
Reflection and transmission spectra allow us to determine the spectral position of the band edge, as well as the energies of the free excitons A and B [2]. One of our samples, having $0.2 \mu \mathrm{m}$ thickness, is thin enough to exhibit excitonic structures in its transmission spectra (figure 1). It will be studied by pump and probe experiments.

It has been shown that the spectral position of the band edge depends on the residual strain in the layer [3]. Four-wave mixing experiments are performed with a $2.4 \mu \mathrm{m}$ sample, which exhibits a shift of $10 \mathrm{meV}$ of its band edge to lower energies.

\section{Non linear luminescence spectra}

At $2.4 \mathrm{~K}$, we study the luminescence spectra when exciting with nanosecond pulses from a $\mathrm{XeCl}$ excimer laser at different excitation intensities (figure 2). The photon energy of the exciting beam is $4.026 \mathrm{eV}$ and its intensity varies from 14 to $116 \mathrm{~kW} . \mathrm{cm}^{-2}$. We fit the emission lines by three contributions of Lorentzian shape. The photon energy of two of them are fixed at 3.480 and $3.486 \mathrm{eV}$. These spectral positions correspond to the radiative recombination of the free excitons $A$ and $B$ respectively. The photon energy of the maximum of the third one depends on the excitation intensity. In figure 3 and figure 4 respectively, we plot the integrated intensity and spectral position of this line (called A) as a function of the intensity of the exciting beam. The figures show two different regions of excitation. For low intensities (less than $50 \mathrm{~kW} / \mathrm{cm}^{2}$ ), the spectral position of the A line does not vary with the excitation intensity, and its integrated intensity tends to saturate. We interpret this emission line as being due to the radiative recombination of bound excitons $\mathrm{I}_{2}$. It saturates when all the impurity sites are occupied by an exciton [4].

For higher excitations, the photon energy of the maximum of the line decreases from 3.4745 to $3.4720 \mathrm{eV}$ and its intensity grows quadratically with the intensity of excitation. Since the lines is located on the low energy side of the bound exciton, we interpret this second behavior as being due to a collision process between free excitons or between bound excitons and electrons or holes. It mean that the bound exciton recombines radiatively and part of its energy is transferred to the other particles during the collision process. The probabilities of this recombination process increases with the density of electron-hole pairs. This explains the quadratic increase of the photoluminescence intensity [5].

\section{Pump and probe experiments}

We perform pump and probe experiments in the nanosecond time regime at $2.4 \mathrm{~K}, 77 \mathrm{~K}$ and $300 \mathrm{~K}$. The pump beam is emitted from a dye laser, pumped optically by the excimer laser. The wavelength of the output laser is tunable in the range from 3.410 to $3.520 \mathrm{eV}$, which covers the excitonic region. The probe beam is obtained from the spectrally broad superradiant emission of a dye, also excited by the excimer laser. Its transmission is focused onto the entrance slit of a spectrometer. We measure the variation of the transmission spectra of the probe beam for different intensities and photon energies of the pump beam (figure 5 and figure 6, respectively). As shown in Figure 5, with increasing intensities of excitation, the transmission curves show a red-shift of about $20 \mathrm{meV}$ of the excitonic absorption edge. This may be due to a band gap renormalisation process which has been previously observed with InGaN samples [6], or simply to a heating of the sample due to the excitation. In addition, the excitonic resonance broadens and vanishes at high intensities of the pump beam. In semiconductors, the exciton energy is generally fixed when increasing the density of carriers. This is due to the compensation of the band gap renormalisation and screening effects. The observed red-shift of the excitonic resonance can be explained by an increase of the lattice temperature about $50 \mathrm{~K}$, induced by the pump beam [7]. Time resolved measurements will be necessary to verify this hypothesis.

Figure 6 shows the transmission of the sample for different photon energies of the exciting pulses. It appears that the processes discussed with figure 5 become effective near the bound exciton region.

Our transmission curves also show a broadening of the excitonic resonance with increasing intensities, which is due to collision processes. At higher intensities of excitation, the excitonic resonance disappears. This is close to the Mott density, which is evaluated to be about $5.910^{18} \mathrm{~cm}^{-3}$ (i.e. an intensity of excitation of $50 \mathrm{~kW} . \mathrm{cm}^{-2}$ ), assuming a lifetime of $16 \mathrm{ps}$ as determined by four wave-mixing, and an optical density of 0.6 . This value is close to that of Ref. [8]. The intensity necessary to induce the Mott transition is found to decrease with increasing temperature of the sample.

\section{Degenerate four-wave mixing experiments}


We perform degenerate four-wave mixing experiments in a standard three-beam configuration, using the third harmonics of a Nd-YAG laser at a repetition rate of $10 \mathrm{~Hz}$. The output pulses have an energy of $3.492 \mathrm{eV}$ and a pulse duration of $20 \mathrm{ps}$. The probe beam is sent through a variable delay line in order to study the diffraction efficiency as a function of the delay between the two pump and the probe beams. The first order of diffraction of the probe beam is sent onto an optical multi-channel analyzer (Reticon camera).

At different temperatures, we study the dynamics of the diffracted beam as a function of the grating period and of the intensity of the pump beams (figure 7). The diffraction efficiency increases quadratically as a function of the intensity of the pump beam, showing no saturation (figure 8). This means that our excitation intensity is well situated inside the $\mathrm{c}^{(3)}$ region of the material and that no higher order processes are present.

Since the time decay is close to our experimental time resolution, we modelize our experiments supposing that the diffraction efficiency is proportional to the square of the time dependent density of carriers. The result of such a deconvolution is shown in figure 9 . The characteristic time is found to be about $16 \mathrm{ps}$. This time is very short, but is of the same order as time constants obtained by time-resolved photoluminescence measurements. It corresponds to the lifetime of the free carriers. No dependence of the characteristic decay on the temperature has been found. This is explained by the fact that our photon energy of excitation is always inducing band to band transitions. Furthermore, the characteristic time does not depend on fringe spacing non on the intensity of the pump beam. This means that carriers are trapped to localized states and do not diffuse considerably into the sample. Such localized states can be due to the columnar growth of the films, which induce structural defects as grain boundaries along the $c$ axis which is perpendicular to the surface [9].

\section{Conclusion}

Our studies demonstrate the important influence of impurities on the optical nonlinearities of Gallium Nitride. Nonlinear luminescence shows saturation of excitons bound to neutral donors, and collision processes at higher intensities of excitation. Pump and probe experiments exhibit a shift of exciton resonance to lower energies as the intensity of the pump beam is increased. This process occurs for pump photon energies higher than the energy of the bound excitons. Pulsed degenerate four-wave mixing experiments at $3.492 \mathrm{eV}$ do not depend on temperature, the fringe spacing and on the intensity of excitation. This means that photo-created excitons are very rapidly localized, within a characteristic time of $16 \mathrm{ps.}$

\section{References}

[1] L. Eckey, J.-Ch. Holst, P. Maxim, R. Heitz, A. Hoffmann, I. Broser , B. K. Meyer, C. Wetzel, E. N. Mokhov, P. G. Baranov , Appl. Phys. Lett. 68, 415-417 (1996).

[2] R. Dingle, D. D. Sell, S. E. Stokowski, M. Ilegems, Phys. Rev. B 4, 1211 (1971).

[3] B Gil, O Briot, RL Aulombard, Phys. Rev. B 52, R17028-17031 (1995).

[4] K Okada, Y Yamada, T Tagushi, F Sasaki, S Kobayashi, T Tani, S Nakamura, G Shinomiya, Jpn. J. Appl. Phys. 35, L787 (1996).

[5] H. Haken, S. Nikitine, Excitons at High Density (Springer-Verlag, Giessen, 1975) .

[6] C.-K. Sun, F. VallÈe, S. Keller, J. E. Bowers, S. P. DenBaars, Appl. Phys. Lett. 70, 2004 (97).

[7] W. Shan, T. J. Schmidt, X. H. Yang, S. J. Hwang, J. J. Song , B. Goldenberg , Appl. Phys. Lett. 66, 985-987 (1995).

[8] Y Kawakami, ZG Peng, Y Narukawa, S Fujita , S Fujita , S Nakamura, Appl. Phys. Lett. 69, 1414-1416 (1996).

[9] Q. Zhu , A. Botchkarev, W. Kim, O. Aktas, A. Salvador, B. Sverdlov, H. Morkoc , S.-C. Y. Tsen, David J. Smith , Appl. Phys. Lett. 68, 1141-1143 (1996). 


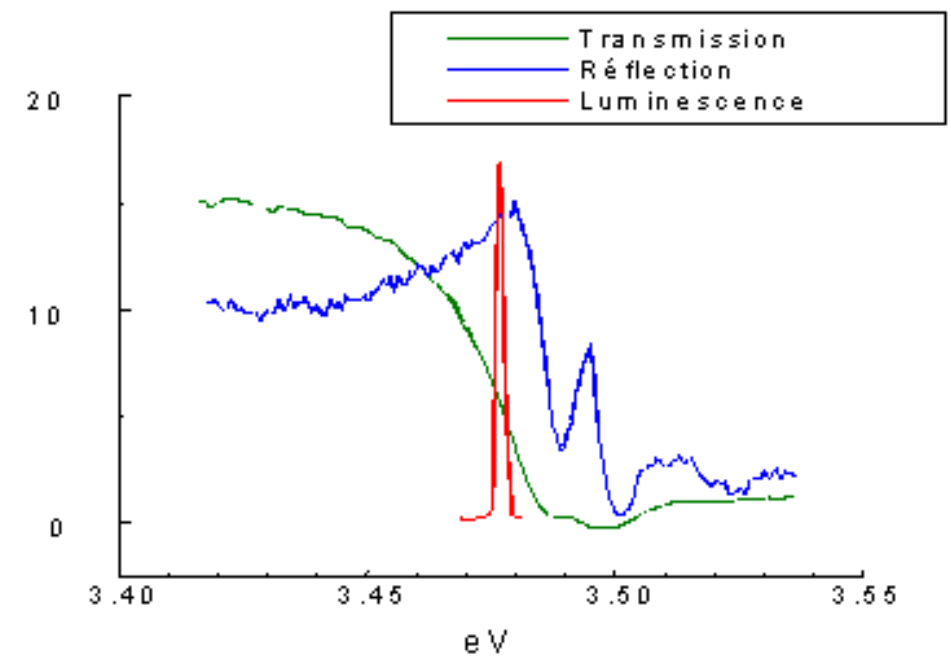

Figure 1. Linear reflection (blue line), transmission (green line) and luminescence (red line) spectra of a $0.2 \mu \mathrm{m}$ thick sample at $2.4 \mathrm{~K}$.

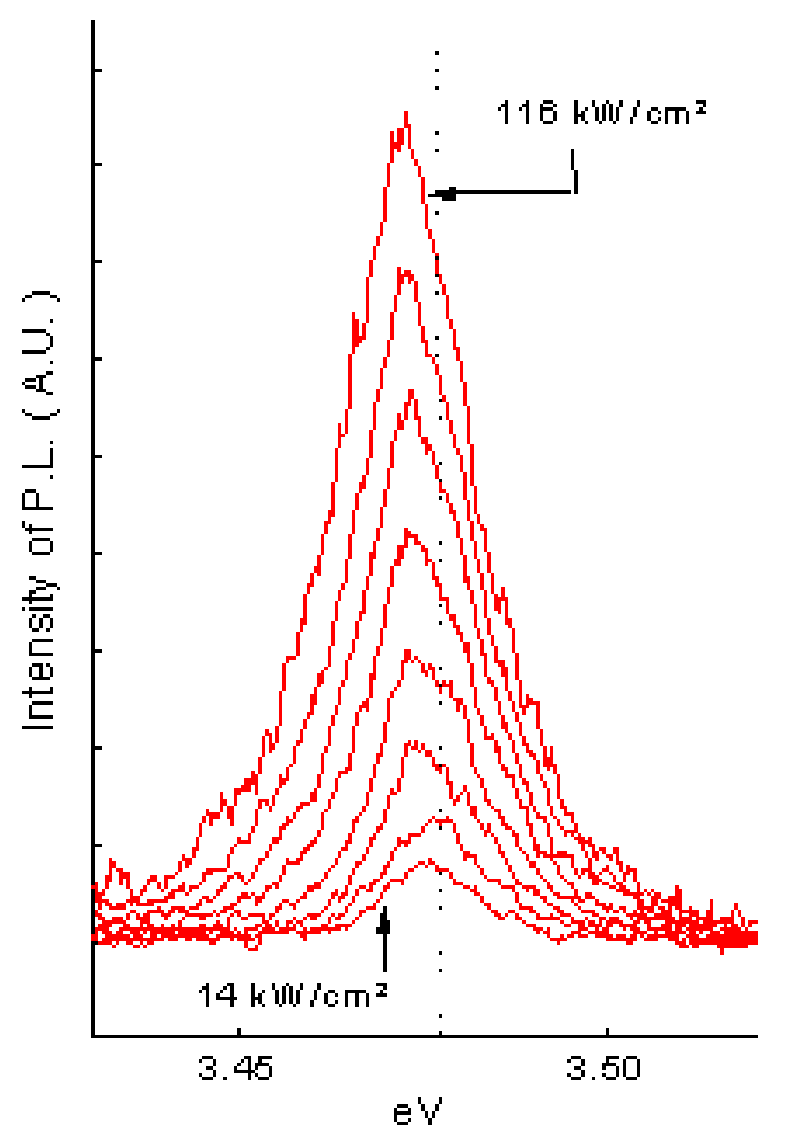

Figure 2. Luminescence spectra at $1.8 \mathrm{~K}$ as a function of photon energy for different intensities of the exciting beam. 


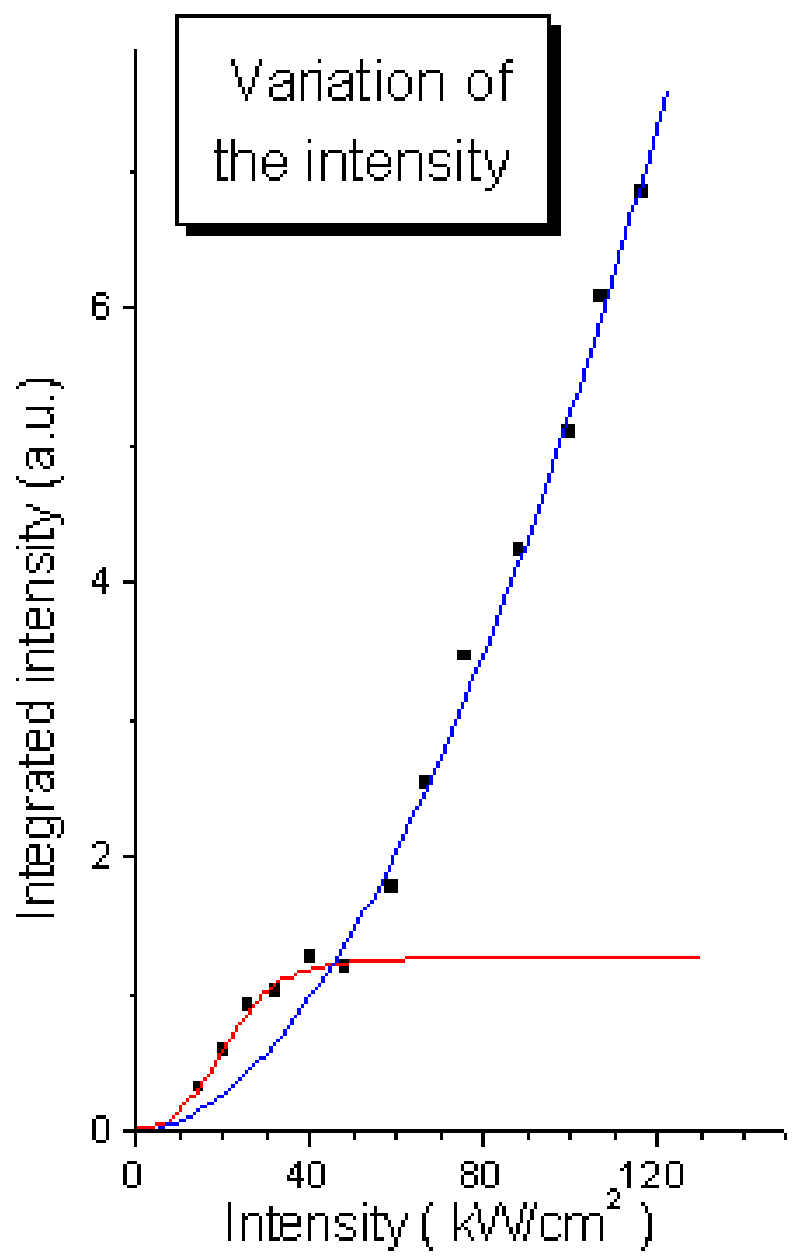

Figure 3. Intensity of the A line as a function of intensity of the pump beam. 


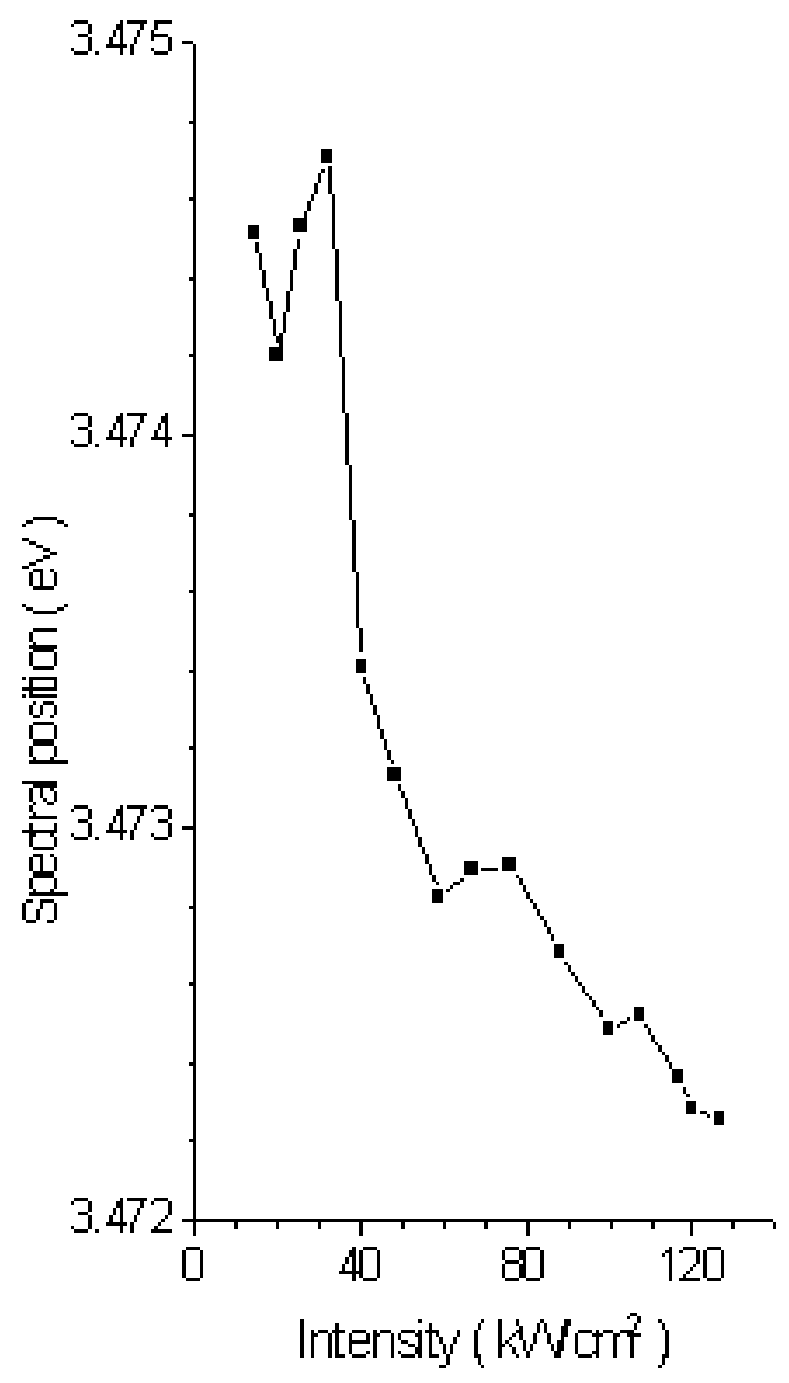

Figure 4. Photon energy of the $A$ line as a function of intensity of the pump beam. 


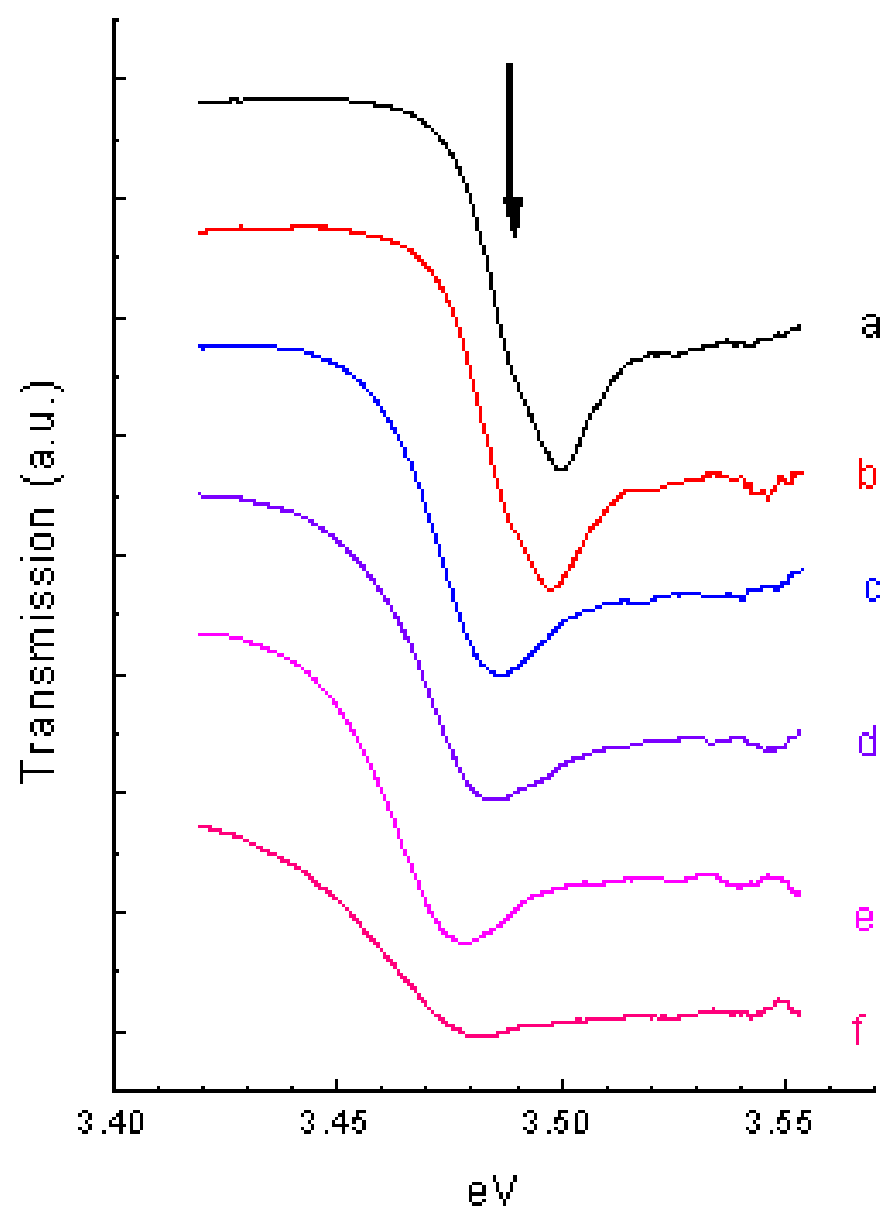

Figure 5. Transmission spectra for different intensities of the pump beam $\left(\mathrm{a}=0 \mathrm{~kW} \cdot \mathrm{cm}^{-2}\right.$, $\mathrm{b}=3 \mathrm{~kW} . \mathrm{cm}^{-2}, \mathrm{c}=19 \mathrm{~kW} . \mathrm{cm}^{-2}, \mathrm{~d}=29 \mathrm{~kW} . \mathrm{cm}^{-2}$, $\left.\mathrm{e}=37 \mathrm{~kW} \cdot \mathrm{cm}^{-2}, \mathrm{f}=62 \mathrm{~kW} \cdot \mathrm{cm}^{-2}\right)$. The photon energy of excitation is $3.484 \mathrm{eV}$.

d 

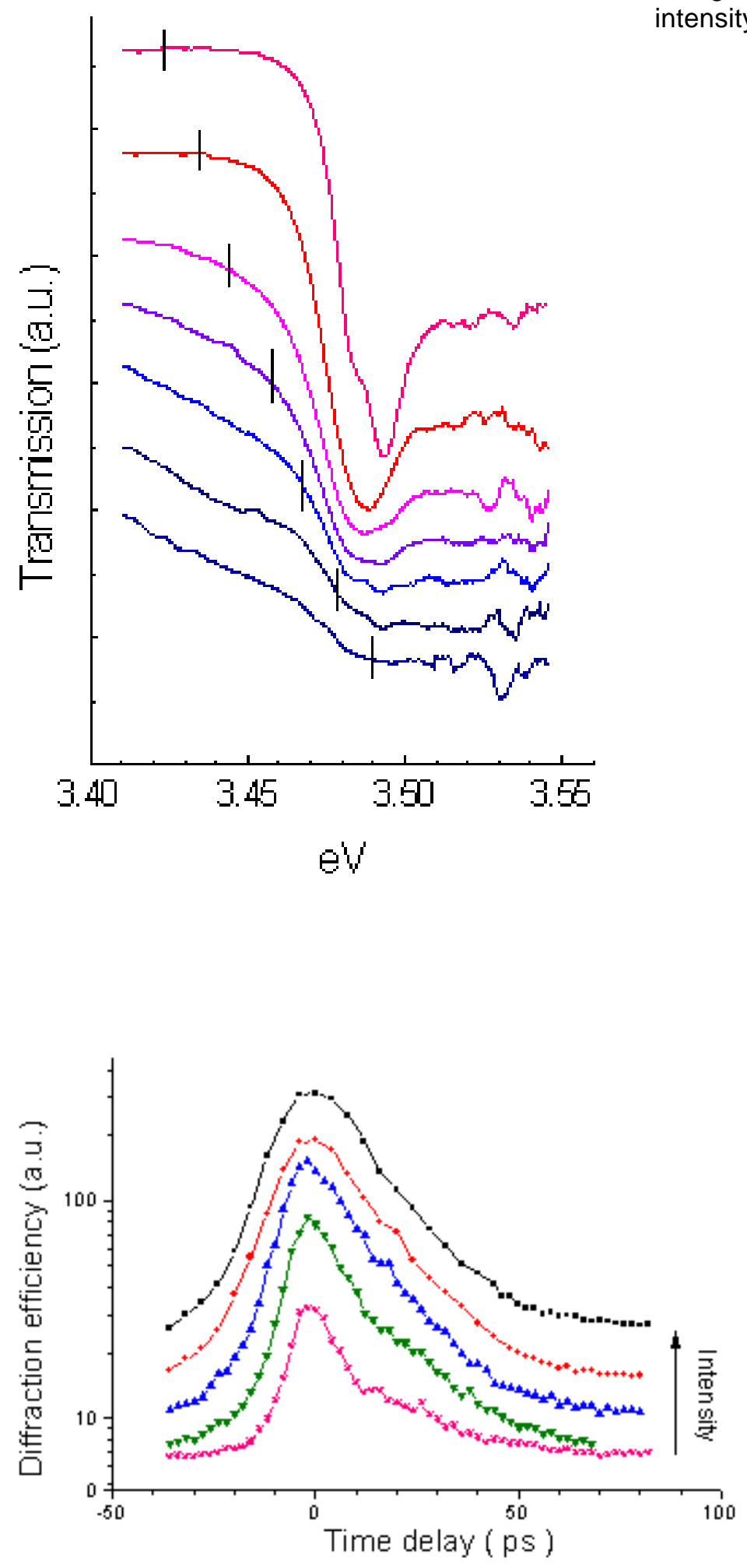

Figure 6. Transmission spectra for different photon energies of the pump beam (indicated by arrows). The intensity of the pump beam is $68 \mathrm{~kW} . \mathrm{cm}^{-2}$.
Figure 7. Variation of the intensity of the diffracted beam for various intensities of the pump beams at $300 \mathrm{~K}$ (squares : $23 \mathrm{~kW} . \mathrm{cm}^{-2}$, circles : $17 \mathrm{~kW} . \mathrm{cm}^{-2}$, up triangles : $15 \mathrm{~kW} . \mathrm{cm}^{-2}$, down triangles : $14 \mathrm{~kW} . \mathrm{cm}^{-2}$, stars : $10 \mathrm{~kW} . \mathrm{cm}^{-2}$ ) 

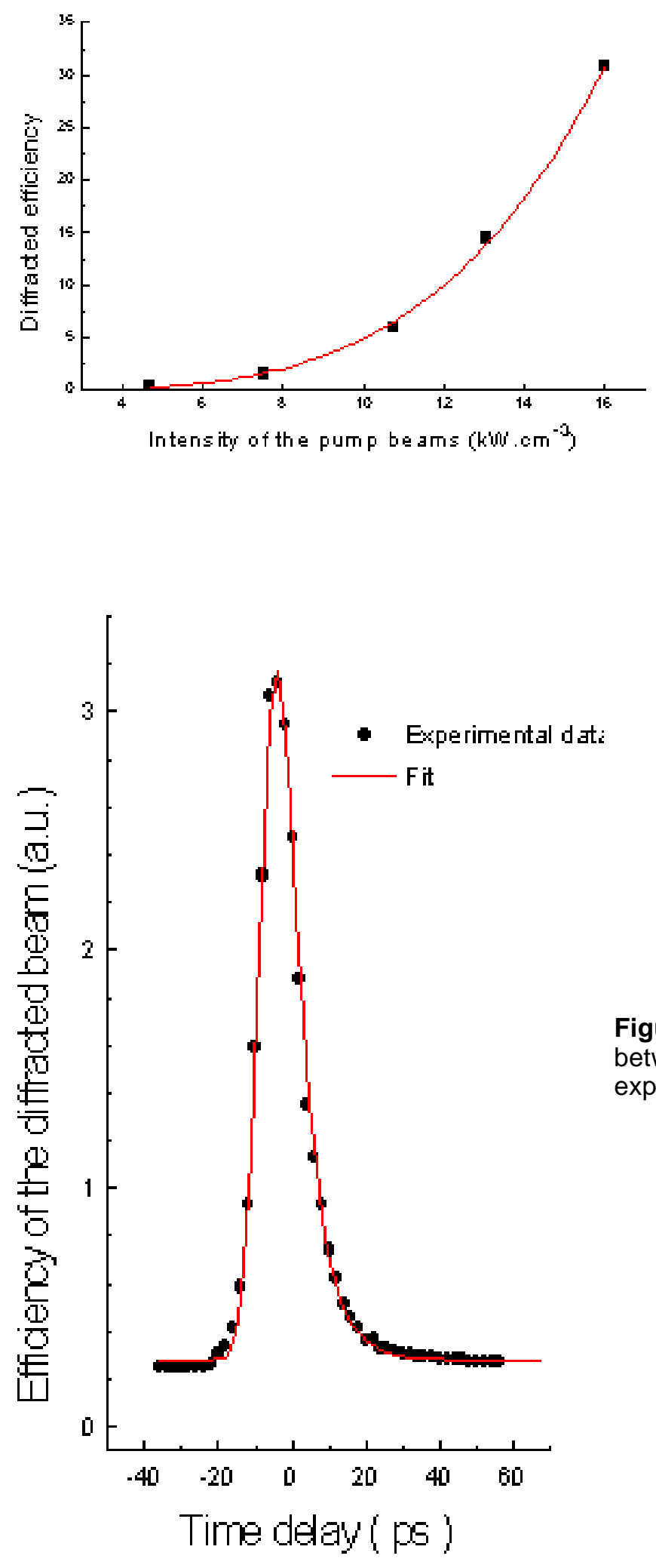

Figure 8. Diffraction efficiency as a function of the intensity of the pump beams.
Figure 9. Signal decay as function of time delay between pump and probe pulses at $300 \mathrm{~K}$ (circles : experimental data, line : fit).

(C) 1997 The Materials Research Society

\begin{tabular}{|l|l|l|l|l}
\hline $\mathrm{M}$ & $\mathrm{R}$ & $\mathrm{S}$ & Internet Journal of & Nitride Semiconductor Research \\
\hline
\end{tabular}

